\title{
EXPERIMENTS ON TRANSVERSE BUNCH COMPRESSION ON THE PRINCETON PAUL TRAP SIMULATOR EXPERIMENT*
}

\author{
E. P. Gilson ${ }^{\dagger}$, M. Chung, R. C. Davidson, M. Dorf, P. C. Efthimion, R. Majeski, E.A. Startsev \\ Plasma Physics Laboratory, Princeton University, Princeton, New Jersey, 08543, USA
}

\begin{abstract}
The Paul Trap Simulator Experiment is a compact laboratory Paul trap that simulates a long, thin chargedparticle bunch coasting through a kilometers-long magnetic alternating-gradient (AG) transport system by putting the physicist in the beam's frame-of-reference. The transverse dynamics of particles in both systems are described by the same sets of equations, including all nonlinear spacecharge effects. The time-dependent quadrupolar electric fields created by the confinement electrodes of a linear Paul trap correspond to the axially-dependent magnetic fields applied in the AG system. Results are presented from experiments in which the lattice period is changed over the course of the experiment to transversely compress a beam with an initial depressed-tune of 0.9. Instantaneous and smooth changes are considered. Emphasis is placed on determining the conditions that minimize the emittance growth and the number of halo particles produced after the beam compression. Envelope equation solutions agree well with the experimental data.
\end{abstract}

\section{INTRODUCTION}

The transverse compression of intense charged-particle beams after acceleration and transport allows for the delivery of large numbers of particles and large amounts of energy to small target spot sizes in applications such as high energy and nuclear physics, ion-beam-driven high energy density physics, heavy ion fusion, and spallation neutron sources [1-6]. The transverse compression should ideally be executed over as few lattice periods of the transport system as possible in order keep the overall system length, and cost, minimized. However, transverse compression cannot be applied so rapidly as to drastically reduce the beam quality, excite collective-mode oscillations, or generate unwanted halo particles [7-11].

The Paul Trap Simulator Experiment (PTSX) is a compact and flexible laboratory facility that simulates the propagation of intense charged-particle beams over thousands of lattice periods through magnetic alternating-gradient (AG) quadrupole transport systems [12-20]. The simulation makes use of the isomorphism between the transverse equations of motion for particles in the two systems [12, 21-23]. In the work described in this paper, the PTSX facility has been used to perform transverse plasma compression experiments in order to develop a better understanding

\footnotetext{
* This research was supported by the U.S. Department of Energy.
}

$\dagger$ egilson@pppl.gov of the physics of transverse compression of intense beams.

The PTSX device is a linear Paul trap [24] confining a one-component plasma of particles with charge $e_{b}$, where the $e_{b} \vec{E}_{\perp}^{\text {ext }}$ forces that the PTSX electrodes exert on the trapped plasma particles are analogous to the $e_{b} \overrightarrow{v_{z}} \times \overrightarrow{B_{\perp}^{e x t}}$ forces that the AG system exert on the beam particles in the beam frame provided that long, coasting beams that are thin relative to the AG system magnet spacing are considered. Specifically, the amplitude and frequency of the voltage waveform applied to the PTSX electrodes correspond to the quadrupole magnet strength and lattice spacing in the AG system. In addition to the equivalence of the applied forces, the self-field forces in both systems can be described by scalar potentials that obey Poisson's equation. In Ref. [12], it was shown that the self-consistent transverse Hamiltonians and the resulting Vlasov equations for the AG system and the PTSX system are equivalent, neglecting end effects. Thus, the very good confinement properties of ions in PTSX and the arbitrary form of the voltage waveform applied to the confining electrodes make PTSX a useful laboratory facility in which to simulate transverse beam compression in an AG system.

\section{PTSX APPARATUS}

As shown in Fig. 1, the PTSX device is a linear Paul

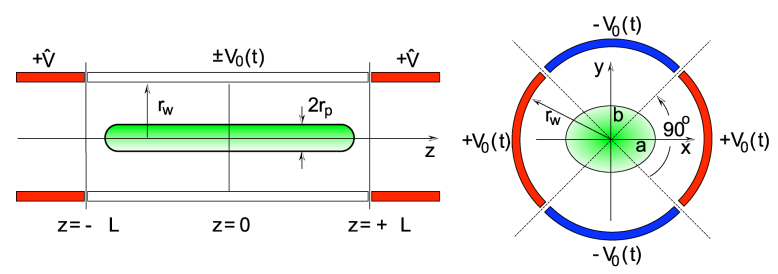

Figure 1: The PTSX device consists of three cylindrical electrodes with radius $r_{w}=0.1 \mathrm{~m}$, each divided into four $90^{\circ}$ sectors. An oscillating voltage $\pm V_{0}(t)$ confines the charge bunch in the transverse plane to a radius $r_{p}$. Static voltages $+\hat{V}$ on the end electrodes confine the ions axially within a length $2 L=2 \mathrm{~m}$.

trap constructed from a $2.8 \mathrm{~m}$-long, $r_{w}=10 \mathrm{~cm}$-radius cylinder. The cylinder is divided into two $40 \mathrm{~cm}$-long end cylinders and a $2 L=2 \mathrm{~m}$-long central cylinder. All cylinders are azimuthally divided into four $90^{\circ}$ segments so that when an oscillating voltage $V_{0}(t)$ is applied with alternating polarity on adjacent segments, the resulting oscillating transverse quadrupole electric field exerts a ponderomotive 
force that confines the plasma radially. To trap the plasma axially, the two end cylinders are biased to a constant voltage $\hat{V}$. Voltage waveforms with amplitudes up to $400 \mathrm{~V}$ and frequencies up to $100 \mathrm{kHz}$ can be used. The trapping voltage is nominally $\hat{V}=36 \mathrm{~V}$. The vacuum pressure of $5 \times 10^{-9}$ Torr prevents neutral collisions from playing an important role in the plasma behavior.

The plasma source is a $1.5 \mathrm{~cm}$-diameter aluminosilicate cesium emitter. Singly-charged cesium ions are extracted by applying a bias of less than 10 volts between the emitter and an acceleration grid. The ions then pass through a separately biased deceleration grid. The ion source is situated in the middle of one of the $40 \mathrm{~cm}$-long cylinders, and to inject a pure cesium ion plasma into the trap, the segments on this $40 \mathrm{~cm}$-long cylinder are temporarily set to oscillate with the voltage $\pm V_{0}(t)$. The injection time $t_{i}$ is several milliseconds in order to allow cesium ions with several $\mathrm{eV}$ of kinetic energy to fill the trap. The injection process is optimized by briefly suppressing ion emission to allow ions to vacate the vicinity of the $40 \mathrm{~cm}$-long trapping electrodes to ensure that the trapped plasma is as quiescent and cool as possible [13].

After trapping the plasma for a time $t_{t}$, that can be up to $300 \mathrm{~ms}$ but is actually $1 \mathrm{~ms}$ for the experimental results presented here, the $40 \mathrm{~cm}$-long cylinder on the opposite end of the PTSX device from the ion source is set to oscillate with voltage $\pm V_{0}(t)$, and the plasma streams out of the trap. Part of the exiting plasma is collected on a moveable 5 $\mathrm{mm}$-diameter collector disk. The inject-trap-dump cycle is repeated to reduce the uncertainty in the data. The collector is moved along a null in the applied potential in the transverse plane in order to collect a radial charge profile of the trapped plasma. The radial density profile is then computed using the measured radial charge profile and knowledge of the area of the collector and the length of the plasma column [14]. Note that since the plasma ions can take several milliseconds to leave the trap, the measurements are necessarily averaged over hundreds of lattice periods.

\section{TRAPPED PLASMA PROPERTIES}

The circular cross section of the PTSX electrodes allows the time-dependent electric potential to be calculated analytically. Near the axis, the potential is quadrupolar and the average smooth focusing frequency [1] of particles' transverse oscillations can be expressed for an applied voltage $V(t)=V_{0 \max } \sin (2 \pi f t)$ as $[1,12]$

$$
\omega_{q}=\frac{8 e_{b} V_{0 \max }}{m_{b} r_{w}^{2} \pi f} \xi,
$$

where $m_{b}=133 \mathrm{amu}$ for $\mathrm{Cs}^{+}$ions in PTSX. The factor $\xi$ depends on the shape of the voltage waveform and $\xi=1 / 2 \sqrt{2} \pi$ for the sinusoidal waveform used herein. Note that $\xi=4 \sqrt{3} /(\eta \sqrt{3-2 \eta})$ for a periodic step-function waveform with fill-factor $\eta$ (the so-called Focusing-Off-Defocusing-Off, or FODO lattice). In addition, the smooth-focusing vacuum phase advance $\sigma_{v}^{s f}$ is given by $\sigma_{v}^{s f}=\omega_{q} / f[1,15,16]$. In order for the particles to be confined radially, the normalized beam intensity $s \equiv \omega_{p}^{2}(0) / 2 \omega_{q}^{2}$ must be less than unity, where $\omega_{p}(0)=\left[n_{b}(0) e_{b}^{2} / m_{b} \epsilon_{0}\right]^{1 / 2}$ is the on-axis plasma frequency. The limit of low $s$ corresponds to the regime where the plasma's space-charge effects are small, while the limit $s \rightarrow 1$ corresponds to the limit of space-charge-dominated beams. For a flat-top density profile, the normalized beam intensity parameter $s$ is related to the depressed tune $\nu / \nu_{o}$ as $\nu / \nu_{0}=\sqrt{1-s}$.

Under quasi-steady-state conditions, for a thermal equilibrium distribution of particles, the average density profile $n_{b}(r)$ is given by $[1,2]$

$$
n_{b}(r)=n_{b}(r=0) \exp \left[-\frac{m_{b} \omega_{q}^{2} r^{2}+2 e_{b} \phi^{s}(r)}{2 k T}\right] .
$$

Here, $k$ is Boltzmann's constant, $T=$ const. is the effective transverse temperature, and the space-charge potential $\phi^{s}(r)$ is determined self-consistently from numerical integration of Poisson's equation $r^{-1} \partial_{r}\left(r \partial_{r} \phi^{s}\right)=$ $-n_{b}(r) e_{b} / \epsilon_{0}$. For $k T \rightarrow 0(s \rightarrow 1)$, the numerator in the exponential must also approach zero in order for the density to remain finite, and this implies a nearly uniform density plasma. In the case of low space-charge density $(s \rightarrow 0)$, the electrostatic potential term in the exponential can be neglected and the radial density profile is nearly Gaussian. As the normalized beam intensity $s$ varies between 0 and 1 , the radial density profile smoothly changes from a flat-top distribution, to a bell-curve shape, and finally to a Gaussian. If $N_{b}=\int_{0}^{r_{w}} n_{b}(r) 2 \pi r d r$ is the line density, and $R_{b}^{2}=\left(1 / N_{b}\right) \int_{0}^{r_{w}} n_{b}(r) 2 \pi r^{3} d r$ is the meansquare radius of the plasma column, then the parameter $\zeta$, defined through the equation $N_{b}=n_{b}(0) \pi R_{b}^{2} \zeta$, is 1 when $s=0$ and $\zeta=2$ when $s=1$. For intermediate values of $s, \zeta$ must be determined from the numerically integrated solutions of Poisson's equation (see Fig. 2).

Integration of Eq. (2) over the radial distribution gives the global radial force balance equation [1]

$$
m_{b} \omega_{q}^{2} R_{b}^{2}=2 k T+\frac{N_{b} e_{b}^{2}}{4 \pi \epsilon_{0}},
$$

which states that the applied confining force must balance both the thermal pressure and the repulsive space-charge force. In the analysis of PTSX results, $R_{b}^{2}$ and $N_{b}$ are calculated as moments of the measured plasma density profiles; $k T$ is the only parameter not known a priori and is inferred from Eq. (3). The transverse emittance $\epsilon_{\perp}$ of a beam is the phase-space area that the beam particle distribution occupies. Although the equivalent emittance of the trapped plasma in PTSX is not directly measured, relative changes in emittance can be inferred by noting that the emittance scales as $\epsilon_{\perp} \propto R_{b} \sqrt{k T\left(N_{b}, R_{b}\right)}$.

\section{TRANSVERSE COMPRESSION}

Transverse compression of the beam can be achieved, in accordance with the global force balance equation 


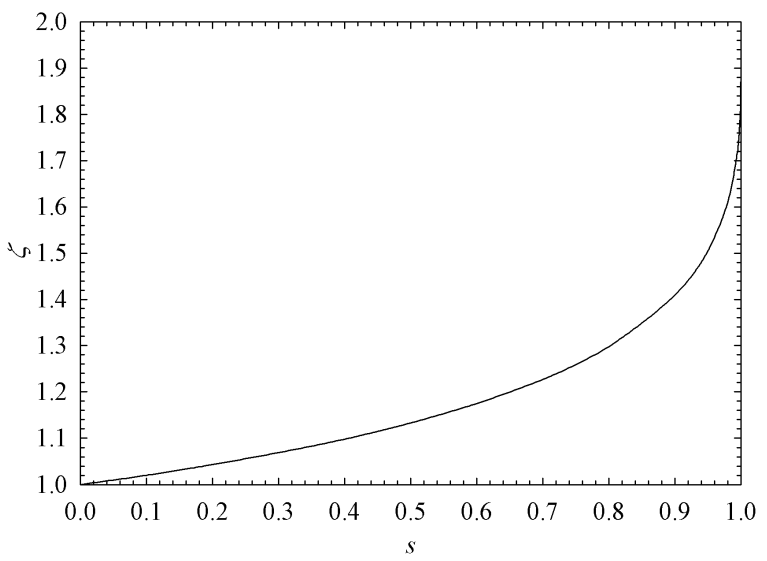

Figure 2: The average radial density profile $n_{b}(r)$ is determined self-consistently from numerical integration of Poisson's equation and Eq. 2. The parameter $\zeta=$ $\left.N_{b} /\left[n_{b}(0) \pi R_{b}^{2}\right)\right]$ is plotted versus the normalized beam intensity parameter $s$. The profile is Gaussian in the limit of weak space-charge, $s \rightarrow 0$ and $\zeta=1$. The profile is uniform when $s \rightarrow 1$ and $\zeta=2$, corresponding to a spacecharge-dominated beam.

[Eq. (3)], by increasing the transverse focusing frequency $\omega_{q}$ using the flexibility of the PTSX device to apply arbitrary voltage waveforms to the transverse confinement electrodes. Assuming that the line density $N_{b}$ does not change during the increase in $\omega_{q}$, the beam will be compressed as long as any increase in the effective perpendicular temperature $k T$ is not too great. Analytical and simulation work by Dorf et al. has explored the effects of adiabatic changes in $\omega_{q}$ on the transverse density profile of the beam [7]. In the present work, the effects of changing $\omega_{q}$ are explored experimentally. The transverse focusing frequency is proportional to $V_{0 \text { max }} f^{-1}$ on PTSX so that there are two experimental parameters that can be changed to implement a variation in $\omega_{q}$. It is expected that only the change in $\omega_{q}$ is relevant, and not whether the change is due to changes in $V_{0 \max }$ or $f$.

Experiments on the transverse compression of plasmas in PTSX due to changes in $V_{0}$ max have been discussed by Chung et al. [17]. Here, experiments in which $f$ is varied in order to adiabatically compress the beam demonstrate that decreases in $f$ are equally well suited as increases in $V_{0 \text { max }}$ for compressing the radial density profile and increasing the peak density of the plasma. A more detailed discussion of transverse beam compression by frequency changes can be found in Ref. [25]. For the plasmas used in these experiments with a normalized beam intensity $s=0.2, R_{b}=0.85 \mathrm{~cm}$., and $k T=0.13 \mathrm{eV}$, the transverse compression may still be considered adiabatic even if the transition is made over only 4 lattice periods. Further, the peak density scales with the transverse focusing frequency as $n_{b}(0) \propto \omega_{q}$ as expected. However, the normalized beam intensity parameter decreases because $s \propto \omega_{q}^{-1}$ due to the dependence of $n_{b}(0)$ on $\omega_{q}$. Instantaneous changes in $\omega_{q}$ may still transversely compress the plasma, but there is substantial emittance growth (transverse heating) associated with this type of transition.

\section{CHANGES IN LATTICE PERIOD $F=1 / T$}

In this section, increases in $\omega_{q}$ created by decreasing the frequency $f=1 / T$ are considered. It is important to note here that voltage waveforms are applied to the PTSX electrodes by generating the waveform $V_{0} \max \sin \phi(t)$, where $\phi$ is an arbitrary function of time, so that once the functional form of the instantaneous frequency $\dot{\phi}(t) / 2 \pi$ is specified, it must be integrated to obtain $\phi(t)$. To obtain a hyperbolic tangent transition of the instantaneous frequency, the required phase function is,

$$
\begin{aligned}
\phi(t)= & 2 \pi \frac{f_{1}+f_{0}}{2} t \\
& +2 \pi \frac{f_{1}-f_{0}}{2} \frac{\tau}{2} \ln \left[\cosh \left(\frac{-\left(t-t_{1 / 2}\right)}{\tau / 2}\right)\right](4)
\end{aligned}
$$

In Fig. 3, the final measured on-axis charge $Q(0)$, which

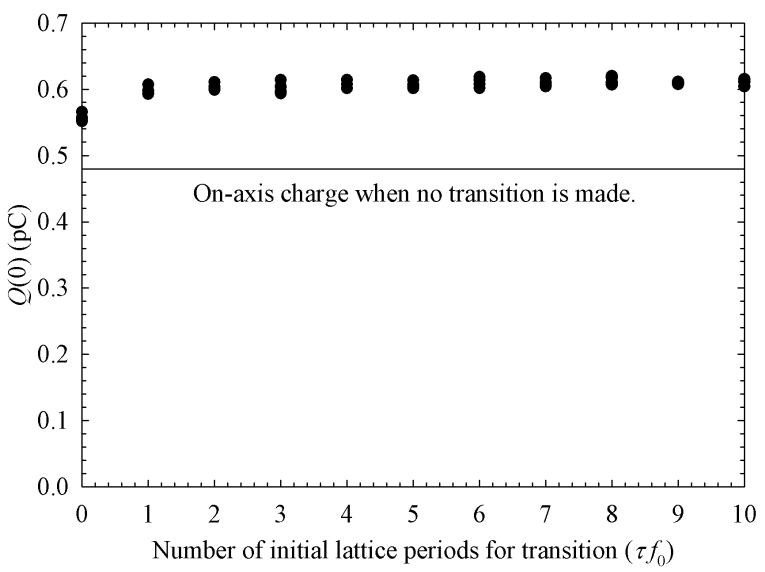

Figure 3: The measured on-axis charge $Q(0)$, which is proportional to the peak plasma density, varies with the number of initial lattice periods over which the increase in $\omega_{q}$ is made. $Q(0)$ increases when the instantaneous frequency $\dot{\phi}(t) / 2 \pi$ is is decreased from $60 \mathrm{khz}$ to $45 \mathrm{kHz}$. An instantaneous change compresses the beam less and increases the emittance as compared to an adiabatic change that is made over two or more lattice periods.

is proportional to the peak plasma density, is plotted versus the number of initial lattice periods over which the increase in $\omega_{q}$ is made. The on-axis charge is $0.48 \mathrm{pC}$ corresponding to normalized beam intensity $s=0.2$, the rms radius is $0.85 \mathrm{~cm}$ and the temperature is $0.13 \mathrm{eV}$ before the charge bunch is compressed. Note that because the smoothfocusing vacuum phase $\sigma_{v}^{s f}$ advance scales like $V_{0 \text { max }}^{1} f^{-2}$, $\sigma_{v}^{s f}$ increases more rapidly when the frequency is changed 
in order to compress the plasma than when the amplitude is changed. Given the operating parameters of the baseline case $\left(V_{0 \max }=150 \mathrm{~V}, f=60 \mathrm{kHz}\right), \omega_{q}$ cannot be increased as much compared to when $V_{0} \max$ is increased, or else $\sigma_{v}^{s f}$ will exceed the single-particle orbit stability limit $\sigma_{v \text { critical }}^{s f}=115.6^{\circ}$ [14]. Therefore, a maximum increase in $\omega_{q}$ of $33 \%$ is used.

An instantaneous decrease $(\tau=0)$ in the frequency from $60 \mathrm{kHz}$ to $45 \mathrm{kHz}$ compresses the plasma and raises the peak density by $17 \%$ while increasing the emittance by $24 \%$. If instead the transition is made over two or more lattice periods, the peak density is raised by $30 \%$ while the emittance does not change discernably. The radial density profiles in Fig. 4 show that an instantaneous change causes

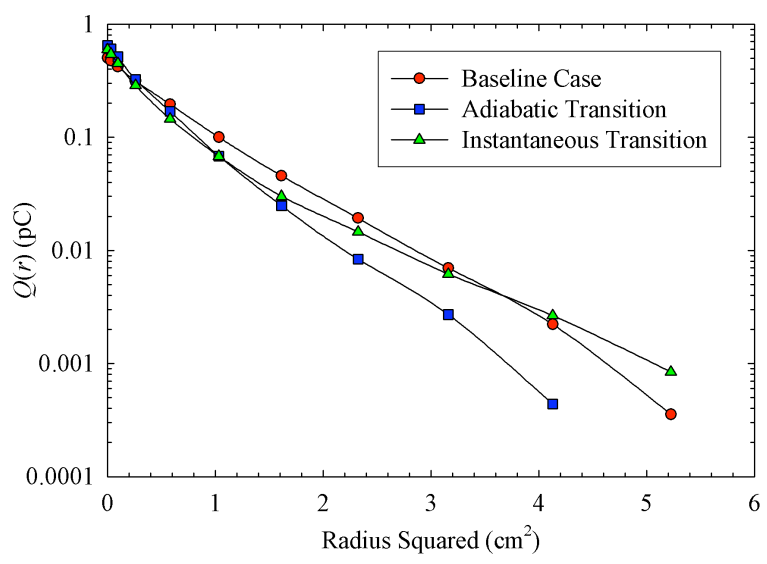

Figure 4: The average radial density profile plotted versus $r^{2}$ is linear when the density profile is Gaussian. Both the baseline case and the adiabatically compressed case have radial density profiles that are Gaussian. In contrast, the instantaneously compressed case has a radial density profile in which some particles have been transferred to a larger radius, thereby producing a super-Gaussian tail.

the creation of a distortion of the original Gaussian radial density profile, corresponding to excitation of a halo particle population. The radial density profile of the plasma after an adiabatic transition remains Gaussian. That the peak density scales linearly with $\omega_{q}$ and that there is minimal emittance growth when $\omega_{q}$ is increased adiabatically agree well with the case where $V_{0} \max$ is changed while $f$ is held fixed. This confirms the notion that $\omega_{q}$ is the important control parameter in characterizing beam compression, as opposed to either $V_{0 \text { max }}$ or $f$ separately. In both cases, the transition may be considered adiabatic when the transition is made over more than about four lattice periods. Note, however, that the change in the smooth-focusing phase advance is different in the two cases.

\section{ENVELOPE EQUATION MODEL COMPARISON}

It is informative to compare the experimental results to the solutions of the coupled transverse envelope equations where the initial condition is taken to be a $\mathrm{KV}$-equivalent matched-beam solution that has the same line density $N_{b}$, rms radius $R_{b}$, and effective transverse temperature $T$ as those measured [1]. This KV-equivalent beam represents a uniform-density charge distribution that has an elliptical cross-section with radii $a$ and $b$ in the transverse $x$ and $y$ directions. If the frequency $f$ is changed instantaneously from $60 \mathrm{kHz}$ to $45 \mathrm{kHz}$, then the mean radius $\sqrt{a b}$ decreases on average, but only by about $8 \%$, and the mean radius exhibits large oscillations as shown in Fig. 5. Further,

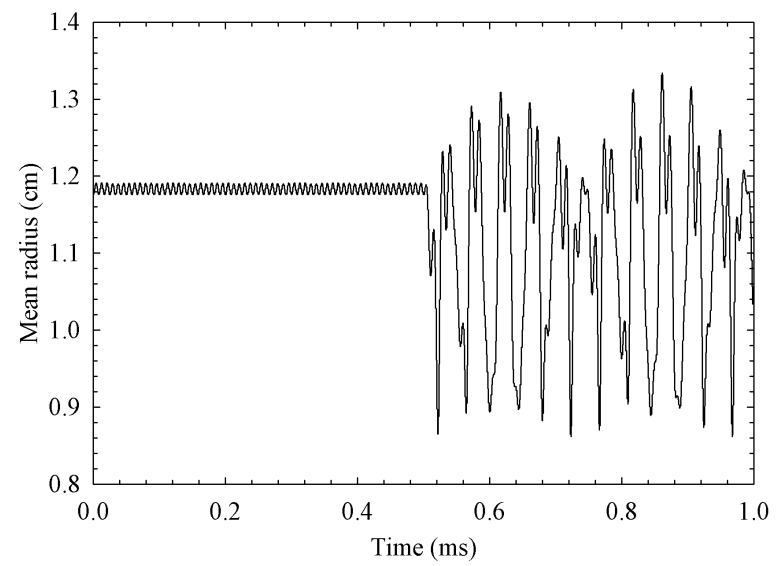

Figure 5: The mean radius $\sqrt{a b}$ undergoes large-amplitude oscillations that do not correspond to a matched-beam solution of the coupled envelope equations when an instantaneous decrease in the frequency $\dot{\phi}(t) / 2 \pi$ from $60 \mathrm{kHz}$ to $45 \mathrm{kHz}$ to compress the charge bunch is made.

the oscillations are not characteristic of a matched-beam solution. Note that the compression agrees well with the observation of a $17 \%$ increase in peak density in the experimental data since $n_{b}(0) R_{b}^{2}=$ const. It is important to note that the emittance was taken to be constant in this calculation despite the fact that the emittance is expected to, and indeed is observed to, increase in the experiment itself.

If the same KV-equivalent beam is then subjected to a transverse compression administered over 5 initial lattice periods, then Fig. 6 shows that the final mean radius is smaller by $12 \%$ which is consistent with the observed increase in peak density of $30 \%$ mentioned above, since $1.12^{2}=1.25$. After the compression, the oscillations in the mean radius are much smaller than in the instantaneouschange case, and the beam remains well matched after the adiabatic transition. 


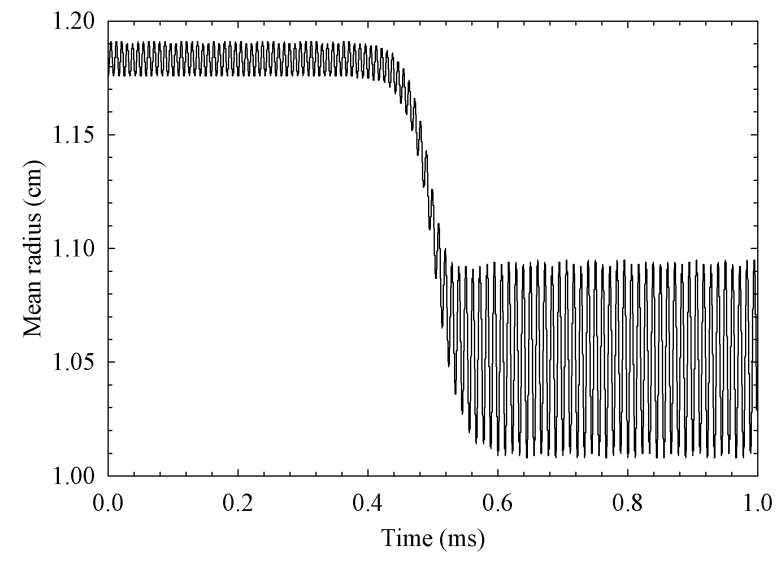

Figure 6: The mean radius $\sqrt{a b}$ decreases as an adiabatic decrease in the frequency $\dot{\phi}(t) / 2 \pi$ from $60 \mathrm{kHz}$ to $45 \mathrm{kHz}$ made over 5 initial lattice periods, $\tau=5 \times(60 \mathrm{kHz})^{-1}$, compresses the charge bunch. The compression is more than in the instantaneous change case, and the mean radius oscillations correspond to a beam that is still well-matched.

\section{CONCLUSIONS}

The transverse compression of a charged particle beam moving through a magnetic alternating-gradient transport system can focus the beam to a small spot size in order to deliver more particle flux and energy to the target. It is important to determine how few lattice periods are needed to make such a transverse compression while maintaining a reasonably well-matched beam envelope. The results of experiments performed with the flexible PTSX facility show that for moderately intense beams with normalized beam intensity parameter $s=0.2\left(\nu / \nu_{0} \sim 0.9\right)$, transverse compression can be affected by changes in the smooth-focusing frequency $\omega_{q}$ over only four lattice periods. The changes in $\omega_{q}$ can be made by using the arbitrary waveform voltage $V(t)$ to change either the field-strength or the lattice periodicity, although the final smooth-focusing vacuum phase advance depends on whether $V_{0}$ max or $f$ is changed.

\section{ACKNOWLEDGEMENTS}

This research was supported by the U.S. Department of Energy.

\section{REFERENCES}

[1] R. C. Davidson and H. Qin, Physics of Intense Charged Particle Beams in High Intensity Accelerators (World Scientific, Singapore, 2001).

[2] M. Reiser, Theory and Design of Charged Particle Beams (Wiley, New York, 1994).

[3] A. W. Chao, Physics of Collective Beam Instabilities in High Energy Accelerators (Wiley, New York, 1993).

[4] P. G. O'Shea, M. Reiser, R. A. Kishek, S. Bernal, H. Li,
M. Pruessner, V. Yun, Y. Cui, W. Zhang, Y. Zou, et al., Nucl. Instrum. and Methods A 464, 646 (2001).

[5] N. Kjærgaard and M. Drewsen, Phys. Plasmas 8, 1371 (2001).

[6] A. B. Sefkow and R. C. Davidson, Phys. Rev. ST Accel. Beams 9, 090101 (2006).

[7] M. Dorf, R. C. Davidson, and E. A. Startsev, Phys. Rev. ST Accel. Beams 9, 034202 (2006).

[8] S. M. Lund and B. Bukh, Phys. Rev. ST Accel. Beams 7, 024801 (2004).

[9] L. K. Spentzouris, J.-F. Ostiguy, and P. L. Colestock, Phys. Rev. Lett. 76, 620 (1996).

[10] D. Neuffer, E. Colton, D. Fitzgerald, T. Hardek, R. Hutson, R. Macek, M. Plum, H. Thiessen, and T.-S. Wang, Nucl. Instrum. and Methods A 321, 1 (1992).

[11] J. Byrd, A. Chao, S. Heifets, M. Minty, T. O. Raubenheimer, J. Seeman, G. Stupakov, J. Thomson, and F. Zimmerman, Phys. Rev. Lett. 79, 79 (1997).

[12] R. C. Davidson, H. Qin, and G. Shvets, Phys. Plasmas 7, 1020 (2000).

[13] M. Chung, E. P. Gilson, M. Dorf, R. C. Davidson, P. C. Efthimion, and R. Majeski, Phys. Rev. ST Accel. Beams 10, 014202 (2007).

[14] E. P. Gilson, R. C. Davidson, P. C. Efthimion, R. Majeski, and E. A. Startsev, American Institute of Physics Conference Proceedings 692, 211 (2003).

[15] E. P. Gilson, R. C. Davidson, P. C. Efthimion, and R. Majeski, Phys. Rev. Lett. 92, 155002 (2004).

[16] E. P. Gilson, R. C. Davidson, P. C. Efthimion, R. Majeski, and H. Qin, in Proceedings of the 2003 Particle Accelerator Conference (IEEE Catalog No. 03CH37423C, 2003), p. 2655.

[17] M. Chung, E. P. Gilson, M. Dorf, R. C. Davidson, P. C. Efthimion, and R. Majeski, Phys. Rev. ST Accel. Beams 10, 064202 (2007).

[18] E. P. Gilson, M. Chung, R. C. Davidson, M. Dorf, P. C. Efthimion, and R. Majeski, Phys. Plasmas 13, 056705 (2006).

[19] E. P. Gilson, M. Chung, R. C. Davidson, M. Dorf, D. Grote, P. C. Efthimion, R. Majeski, and E. A. Startsev, Nucl. Instrum. and Methods A 577, 117 (2007).

[20] E. P. Gilson, M. Chung, R. C. Davidson, P. C. Efthimion, R. Majeski, and E. A. Startsev, Nucl. Instrum. and Methods A 544, 171 (2005).

[21] H. Okamoto and H. Tanaka, Nucl. Instrum. and Methods A 437, 178 (1999).

[22] N. Kjærgaard, K. Mølhave, and M. Drewsen, Phys. Rev. E 66, 015401 (2002).

[23] R. Takai, H. Enokizono, K. Ito, Y. Mizuno, K. Okabe, and H. Okamoto, Jap. J. Appl. Phys. 45, 5332 (2006).

[24] W. Paul and H. Steinwedel, Z. Naturforsch. A 8, 448 (1953).

[25] E. P. Gilson, M. Chung, R. C. Davidson, P. C. Efthimion, and R. Majeski, Phys. Rev. ST Accel. Beams, submitted for publication. 